\title{
Prospects of Hybrid Recording Materials
}

\author{
N. Inaba and N. Ota \\ R \& D Division, Hitachi Maxell Ltd., 6-20-1 Kinunodai, Yawara-mura, Tsukuba-gun, Ibaraki 300-2496
}

Thermally assisted or hybrid magnetic recording system is expected to be a data storage device with high areal density beyond $1 \mathrm{~Tb} / \mathrm{in}^{2}$. Several features of the hybrid recording are treated, such as high resolution recording capability by sharp thermal gradient, and pico-second order high-speed magnetization reversal. Since the hybrid recording solves the head field limit problem, the high magnetic anisotropy $\left(K_{\mathrm{u}}\right)$ materials with order of $10^{7}$ $\mathrm{erg} / \mathrm{cm}^{3}$ are used for the hybrid recording media. We discuss the candidates of the high $K_{\mathrm{u}}$ materials, FePt and $\mathrm{CoPt} \mathrm{L1}_{0}$ ordered alloy, $\mathrm{Tb}-\mathrm{Fe}-\mathrm{Co}$ amorphous, and $\mathrm{Co} / \mathrm{Pd}$ multilayer thin films.

Key words: thermally assisted magnetic recording, hybrid recording, $\mathrm{Ll}_{0}$ ordered alloy, $\mathrm{FePt}, \mathrm{CoPt}, \mathrm{Tb}-\mathrm{Fe}-\mathrm{Co}$, $\mathrm{Co} / \mathrm{Pd}, \mathrm{Co} / \mathrm{Pt}$ multilayer thin film, magneto anisotropy

\section{Introduction}

Magnetic recording system has been required with high areal density and high data rate recording. Commercial hard disk drives with areal densities around $60 \mathrm{~Gb} / \mathrm{in}^{2}$ are available today, and areal densities beyond $100 \mathrm{~Gb} / \mathrm{in}^{2}$ have been demonstrated at a laboratory level. Moreover, the feasibility of perpendicular magnetic recording system at a density of $1 \mathrm{~Tb} / \mathrm{in}^{2}$ has been studied by various authors ${ }^{1)-5}$. With increasing the areal density, the medium noise should be decreased in order to maintain the sufficient signal-to-noise-ratio (SNR) to replay. Since the medium noise depends on a switching volume for magnetization reversal in the media, the increase of the recording density has been traditionally approached by reducing a medium grain size and enhancing the isolation of the magnetic grains in order to break exchange coupling. This approach, however, leads to the thermal stability problem of the recorded bits, when the magnetic anisotropy energy per switching volume $K_{\mathrm{u}} V$ become comparable with the thermal energy $\mathrm{k}_{\mathrm{B}} T$. Under such conditions, the medium starts to exhibit the superparamagnetic behavior. To preserve the recorded bits for a period of ten years without the thermal decay, the stability ratio $K_{\mathrm{u}} V / \mathrm{k}_{\mathrm{B}} T$ is required to be more than $50^{6)-8 \text { ) }}$. Here, $K_{\mathrm{u}}$ is the magnetic anisotropy constant, $V$ is magnetic switching volume, $\mathrm{k}_{\mathrm{B}}$ is the Boltzmann constant, and $T$ is the absolute temperature, respectively. As the grain volume is reduced with higher areal density, the higher $K_{\mathrm{u}}$ materials are needed to maintain the stability.

Recently, various extremely high $K_{\mathrm{u}}$ materials, such as FePt ordered alloy with the $\mathrm{L}_{0}$ phase structure ${ }^{9)}$, have been investigated as the high density recording media with the thermal stability. These materials have the $K_{\mathrm{u}}$ values of the order of $10^{7} \mathrm{erg} / \mathrm{cm}^{3}$, which are more than 10 times as large as those of the conventional $\mathrm{Co}-\mathrm{Cr}-\mathrm{Pt}$ media ${ }^{10)}$. Increasing $K_{\mathrm{u}}$, however, also increases the dynamic coercivity or the medium switching field $H_{0}$ at writing frequency $\left(\square 1 \mathrm{GHz}\right.$ ), since the $H_{0}$ is proportional to the ratio $K_{\mathrm{u}} / M_{\mathrm{s}}\left(M_{\mathrm{s}}=\right.$ saturation magnetization). The high $K_{\mathrm{u}}$ media would consequently have very high $H_{0}$ greater than $20 \mathrm{kOe}$, which is far in excess of available write field capability of the head $(\square 18 \mathrm{kOe})^{11)}$. The head field capability limits the solution of the thermal stability problem by increasing the anisotropy energy, and probably limits the potential areal density of the conventional magnetic recording system, as shown in Fig. 1 (a).

One approach to solving the above problems in the conventional magnetic recording system is a thermally assisted magnetic recording, or a hybrid magnetic recording. Recently, the theoretical and the experimental researches ${ }^{12)-17)}$ have been reported. This paper attempts to provide an overview of the hybrid recording system. Specially, we will discuss the high $K_{\mathrm{u}}$ material candidates for the hybrid recording media. (a) Magnetic Recording

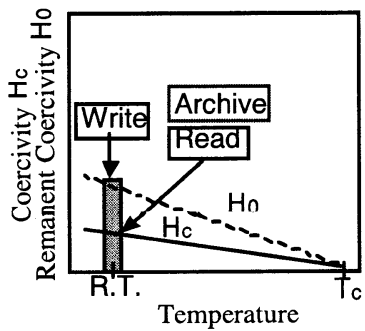

(b) Hybrid Recording

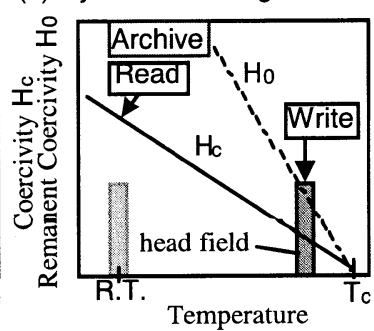

Fig.1 Principle of hybrid recording. (a) conventional magnetic recording, (b) hybrid recording.

\section{Characteristics of hybrid recording system}

\subsection{Merits of hybrid recording}

The hybrid recording is the combined technology of the magnetic and the optical magnetic recording, and its concept is very simple as shown in Fig. 1 (b). The high coercivity media are heated locally during the writing process. Heating reduces the switching field, so that data can be recorded by the available writing head fields. After writing, the media should cool down quickly to room temperature. The recorded data is archived at room temperature, where the coercivity is much larger than the head field. The data is played back by a high sensitive 
magneto flux detector, such as a giant magneto-resistive (GMR) head

In the magnetic recording system, the bit transitions are determined by the magnetic field from the writing head, while the transitions are determined by the characteristics of the thermal profile in the magneto optical recording media. The hybrid recording process has both the thermal profile in the media and the writing head field. Because of this, the hybrid recording methods can be classified roughly into three ways ${ }^{12)}$ to record the bits: (i) track width of writing head $\left(T_{\mathrm{w}}\right)<$ heating spot size $\left(D_{\mathrm{hs}}\right)$, (ii) $T_{\mathrm{w}}>D_{\mathrm{hs}}$, and (iii) $T_{\mathrm{w}}, \quad D_{\mathrm{hs}}$. The first type method $\left(T_{\mathrm{w}}<D_{\mathrm{hs}}\right)$ is very similar to conventional magnetic recording because the transitions and the track width are determined by the magnetic field of the writing head and the medium is only heated to reduce the coercivity. The sharpness of the transitions depends on the field gradient from the head. The second type method $\left(T_{\mathrm{w}}>D_{\mathrm{hs}}\right)$ is similar to the magneto optical recording since the transitions and track width are determined by the characteristics of the thermal profile. In the third type method ( $\left.T_{\mathrm{w}}, D_{\mathrm{hs}}\right)$, the bits are determined by both the magnetic field profile and the thermal profile. The sharpness of the transitions probably depends on the field gradient from the head, the medium temperature profile during the writing process, and the medium characteristics during the cooling process to room temperature after recording.

The track width and the thickness of the main pole for the $1 \mathrm{~Tb} / \mathrm{in}^{2}$ single pole type (SPT) write head are estimated to be $38 \mathrm{~nm}$ and $50 \mathrm{~nm}$, respectively ${ }^{11)}$. The spot size of the focused blue laser light $(\lambda=405 \mathrm{~nm})$ is about $500 \mathrm{~nm}$, which is about 10 times larger than the pole size of the $1 \mathrm{~Tb} / \mathrm{in}^{2}$ SPT head. The spot size is larger than the track width of the present commercial hard disk drives ( $\square$ $300 \mathrm{~nm})$. So, the first type method is probably one approach to realize the hybrid recording. This method needs the low noise media with the exchange-decoupled magnetic grains, since small bit patterns are written by the sharp head field gradient and the heating process is only used for reducing the medium coercivity.

There is a possibility of recording tiny bits by the sharp thermal gradient. Figure 2 shows the magnetic force microscope (MFM) images of the domains recorded on the $\mathrm{Tb}-\mathrm{Fe}-\mathrm{Co}$ specimen by the magnetic recording and the second type hybrid recording methods ${ }^{18)}$. The specimen is the perpendicular magnetic recording medium with the coercivity $H_{\mathrm{c}}$ of $3 \mathrm{kOe}$ at room temperature. When the 100 kFCI bit patterns (bit length : $250 \mathrm{~nm}$ ) were written using a conventional thin film head (head field $>H_{\mathrm{c}}$ ) without heating, the clear domain patterns could not be observed, as shown in Fig. 2 (a). On the other hand, as shown in Fig. 2 (b), the $250 \mathrm{kFCI}$ bit patterns (bit length : $100 \mathrm{~nm}$ ) could be recorded, when the focused pulse laser was irradiated to the medium locally among an uniform magnetic field of 200 Oe. The $20 \mathrm{~nm}$-length tiny magnetic domains in a $\mathrm{Tb}-\mathrm{Fe}-\mathrm{Co} \mathrm{MO}$ recording media, as mentioned later, can be recorded by the sharp thermal gradient ${ }^{24)}$. These results suggest that the bit transitions of the strong exchange coupling media can be determined by the thermal profile. When a tiny heat source is developed and an areal density of the pinning sites in the media is increased, the smaller bit patterns will be able to be recorded by the sharp thermal gradient. (a) Magnetic recording

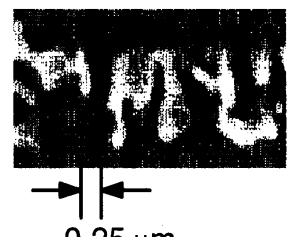

$0.25 \mu \mathrm{m}$

(100 kFCI writing condition)

- $\mathrm{Hex}_{\mathrm{e}}>\mathrm{H}_{\mathrm{c}}(=3 \mathrm{kOe})$ (b) Hybrid recording

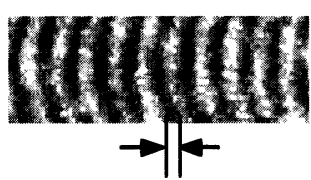

$0.1 \mu \mathrm{m}$

(250 kFCI writing condition)

- Hex $200 \mathrm{Oe}$

- PLaser $=5.5 \mathrm{~mW}$

wavelength $=650 \mathrm{~nm}$
Fig.2 Magnetic force microscope images of domain patterns recorded by (a) magnetic recording method, and (b) hybrid recording method ${ }^{18)}$.

\subsection{Possibility of high speed recording}

The data storage system is required with the high speed recording as well as the high areal density recording. Switching time of magnetization reversal in the media is important factor affecting the high speed recording, or the high frequency recording. When the magnetic field is applied with the frequency range over $10^{9} \mathrm{~Hz}$, every magnetic material faces the switching speed limit caused by natural resonance. The switching time strongly depends on Gilbert's damping constant $\alpha$ for the material ${ }^{19)}$. The switching time of the conventional magnetic recording media is estimated to be larger than 1 ns by numerical micromagnetic simulation ${ }^{20), 21)}$, since the $\alpha$ for the $\mathrm{Co}-\mathrm{Cr}$ alloy medium materials are indicated to have small values around 0.01 by the ferromagnetic resonance experiments ${ }^{22)}$. The recording process of the hybrid recording system might spend longer switching time than that of the conventional magnetic recording, since the increase of the medium temperature by heating is due to the phonon oscillation.

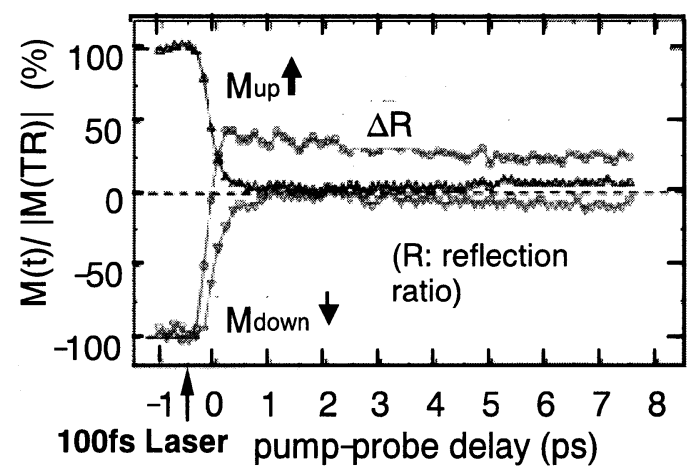

Fig.3 Pico-second order magnetization reversal induced by 100 femto-second laser irradiation ${ }^{23)}$.

As shown in Fig. 3, a pico-second order magnetization reversal is observed in the $\mathrm{Gd}-\mathrm{Fe}-\mathrm{Co}$ perpendicular thin film, when a 100 femto-second laser is irradiated on the 
film ${ }^{23)}$. This switching speed is much faster than the ferromagnetic resonance frequency. The high speed spin reversal of the $\mathrm{Gd}-\mathrm{Fe}-\mathrm{Co}$ induced by femto-second laser pulse is due to the direct photon-electron interaction. This result suggests that the hybrid recording system will overcome the ferromagnetic resonance limit as well as the paramagnetic limit.

\section{Material candidates for hybrid recording media}

\subsection{High $K_{\mathrm{u}}$ materials}

The bit size of the $1 \mathrm{~Tb} / \mathrm{in}^{2}$ is roughly $15 \times 40 \mathrm{~nm}^{2}$. In order to keep medium noise within acceptable bounds, each bit contains only 20-30 magnetic grains with grain sizes on the order of $5 \mathrm{~nm}$, which is about one-half that of conventional media. When the thermal stability factor of the grain should be more than 50 at room temperature, the anisotropy energy of the grain is required to be more than $2 \times 10^{7} \mathrm{erg} / \mathrm{cm}^{3}$.

Typical high $K_{\mathrm{u}}$ materials are plotted in the schematic picture of Fig. 4 in order to demonstrate the characteristics of the materials. The horizontal direction shows the intensity of the exchange coupling, while the vertical axis shows the crystallized structure. The contour lines show the intensity of the anisotropy energy. The typical permanent magneto materials, such as FePt and $\mathrm{CoPt} \mathrm{L}_{0}$ ordered alloy, $\mathrm{Nd}-\mathrm{Fe}-\mathrm{B}$, and $\mathrm{SmCo}_{5}$, have high $K_{\mathrm{u}}$ values more than $10^{7} \mathrm{erg} / \mathrm{cm}^{3}$. The $\mathrm{Co} / \mathrm{Pd}$ multilayer thin films and the $\mathrm{Tb}-\mathrm{Fe}-\mathrm{Co}$ amorphous thin films have also the high perpendicular anisotropy enegy, and these materials are convenient to the magneto optical recording media. Those high $K_{\mathrm{u}}$ materials shown in Fig. 4, however, take strong exchange coupling, which is the origin of the medium noise in the conventional magnetic recording. So, the exchange coupling is a important factor to use these high $K_{\mathrm{u}}$ materials for the high density recording media.

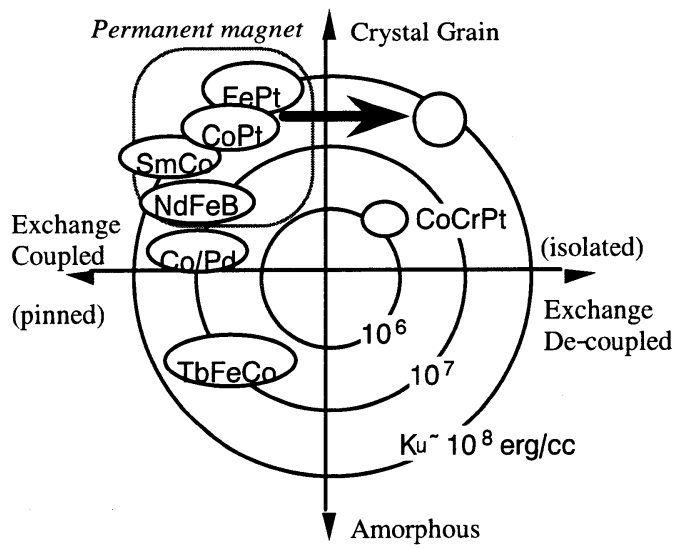

Fig.4 Candidates of hybrid recording materials.

\section{2 $\mathrm{Tb}-\mathrm{Fe}-\mathrm{Co}$ amorphous materials}

$\mathrm{Tb}-\mathrm{Fe}-\mathrm{Co}$ thin films are perpendicular anisotropy amorphous films with strong exchange coupling, which are typically used for the magneto optical recording media. The exchange coupling is disadvantage to the conventional magnetic recording media, since it is difficult to reduce the cluster size of the magnetization reversal. On the other hand, as mentioned above, the bits can be written in the $\mathrm{Te}-\mathrm{Fe}-\mathrm{Co}$ media by the thermally assisted recording. Figure 5 shows the MO signals for the isolated domains recorded with the length of $20 \mathrm{~nm}^{24)}$. The signals were read out by using a magnetic amplifying MO system. This result suggested that the high linear density over 1000 $\mathrm{kFCI}$ can be recorded in the $\mathrm{Tb}-\mathrm{Fe}-\mathrm{Co}$ media by using thermally assisted recording. Kojima et $\mathrm{al}^{25)}$ reported that the possibility of the high track density of 100 kTPI was demonstrated by using the thermal gradient of the heating spot, and also the recorded bit size depended strongly on the underlayer structure. In order to write a small bit in the $\mathrm{Tb}-\mathrm{Fe}-\mathrm{Co}$ media with the strong exchange coupling, it is important to increase the areal density of the wall pinning sites and the wall coercivity.

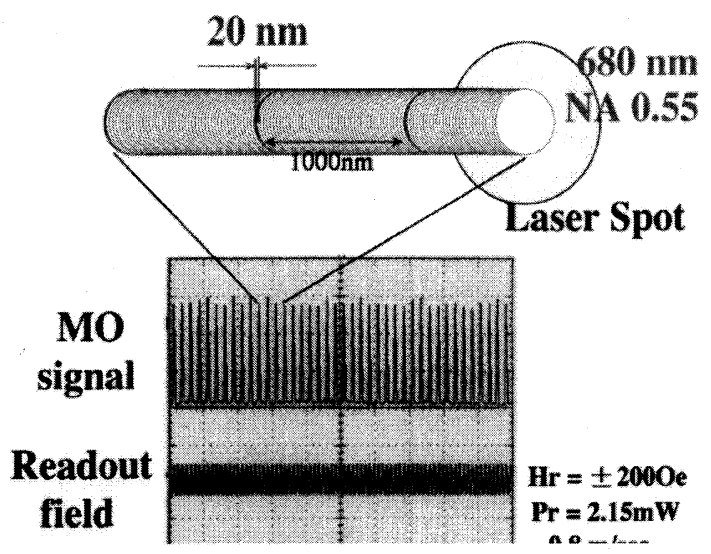

Fig.5 Magneto optical signals from isolated patterns with $20 \mathrm{~nm}$ bit length recorded by thermally assisted recording ${ }^{24)}$.

\subsection{Co/Pt and $\mathrm{Co} / \mathrm{Pd}$ multi-layer thin films}

$\mathrm{Co} / \mathrm{Pt}$ and $\mathrm{Co} / \mathrm{Pd}$ multilayer thin films have large perpendicular magnetic anisotropy $K_{\mathrm{p}}$ and large polar Kerr effect, which are convenient for magneto-optical recording media. Recently, these films have been also actively investigated for the perpendicular magnetic recording media because of their high $K_{\mathrm{p}}$. Curie temperature $T_{\mathrm{c}}$ as well as the $K_{\mathrm{p}}$ is important factor to apply the multilayer films to the hybrid recording media.

Magnetic properties of the multilayer films depend on the thickness of the Co, Pt, and Pd layers. The $K_{\mathrm{p}}$ values depend on both the Co layer and the $\mathrm{Pt}$ (or $\mathrm{Pd}$ ) layer thickness, since the $K_{\mathrm{p}}$ is induced by the lattice strain at the interface between the Co and the Pt (or Pd) layers. The $T_{\mathrm{c}}$ depends strongly on the Co layer thickness ${ }^{26)}$, and weakly on the $\mathrm{Pt}$ (or $\mathrm{Pd}$ ) layer thickness ${ }^{27)}$. Co/Pd multilayer specimens with different Co layer thickness were prepared by a dc magnetron sputtering deposition with a low Ar gas pressure of $0.3 \mathrm{~Pa}$. The Co layer thickness $t_{\mathrm{Co}}$ was changed from $0.2 \mathrm{~nm}$ to $0.5 \mathrm{~nm}$, while the Pd layer thickness was constant $(0.8 \mathrm{~nm})$. With increasing the Co layer thickness, the $M_{\mathrm{s}}$ increases linearly from $510 \mathrm{emu} / \mathrm{cc}$ to $710 \mathrm{emu} / \mathrm{cc}$, while the $K_{\mathrm{p}}$ has a maximum value of $6 \times 10^{6} \mathrm{erg} / \mathrm{cc}$ around $t_{\mathrm{Co}}=0.3 \sim 0.4 \mathrm{~nm}$. The $M_{\mathrm{s}}$ of these specimens 
decrease monotonically with increasing temperature, as shown in Fig. 6. The decreasing rate of the $M_{\mathrm{s}}$ is larger. when the $t_{\text {Co }}$ become thiner. The $K_{\mathrm{p}}$ values also decrease monotonically with increasing the temperature. The $K_{\mathrm{p}}$ of the thiner Co-layer specimen decreases rapidly. For instance, the $K_{\mathrm{p}}$ value of the $0.2 \mathrm{~nm}$-thick specimen disappears at a temperature around $500 \mathrm{~K}$, where the $M_{\mathrm{s}}$ value vanishes. These results suggest that the $T_{\mathrm{c}}$ of the $\mathrm{Co} / \mathrm{Pd}$ multilayer films decreases with decreasing the $\mathrm{Co}$ layer thickness.

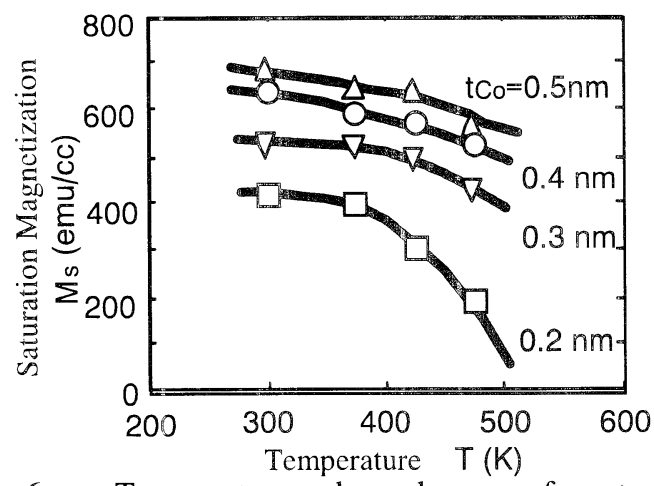

Fig.6 Termperature dependence of saturation magnetization $M_{\mathrm{s}}$ for $\mathrm{Co} / \mathrm{Pd}$ multilayer specimens.
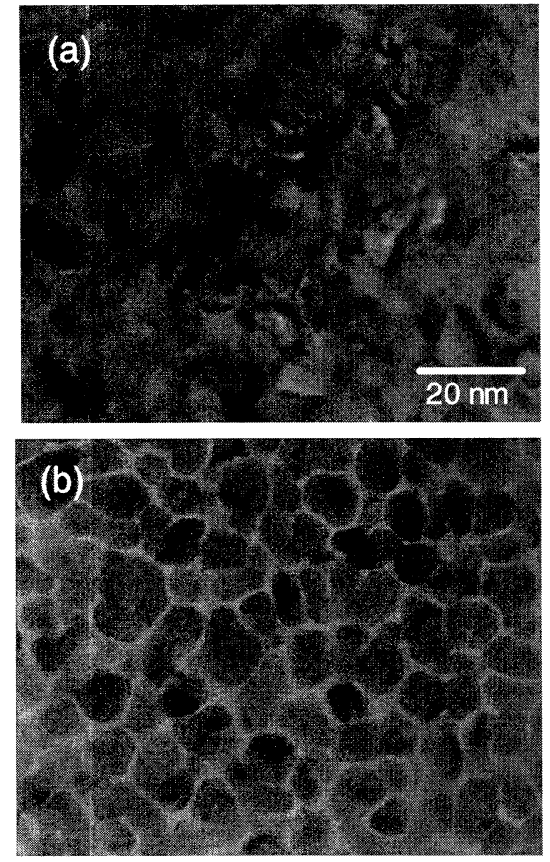

Fig.7 Plan-view transmission electron microscope (TEM) images of $\mathrm{Co} / \mathrm{Pd}$ multilayer specimens deposited with different Ar pressure conditions. (a) low pressure $(0.3 \mathrm{~Pa})$, (b) high pressure $(11 \mathrm{~Pa})$.

The magnetic properties of the multilayer films depend on the film structure as well as the $\mathrm{Co}, \mathrm{Pd}$ (or Pt) layer thickness. Figure 7 shows the plan-view transmission electron microscope (TEM) images of the multilayer films deposited in the different Ar pressure conditions. The specimen with a low pressure $(0.3 \mathrm{~Pa})$ deposition is a continuous medium with the strong exchange coupling, while the high pressure $(11 \mathrm{~Pa})$ specimen become an exchange-decoupled medium with the isolated magnetic grains, Figure 8 shows the temperature dependence of $M_{\mathrm{s}}$ for these two specimens. The $M_{\mathrm{s}}$ values of the high pressure specimen decreases quickly with increasing the temperature, though the Co layer thickness is the same value of $0.4 \mathrm{~nm}$. These results suggest that the Curie temperature decreases with isolating the magnetic grains in the media.

Since the writing process in the hybrid recording system will be probably carried out at the temperature range around $500 \mathrm{~K} \quad 600 \mathrm{~K}$, the $\mathrm{Co} / \mathrm{Pd}$ multilayer films with $\mathrm{t}_{\mathrm{Co}}=0.3 \quad 0.4 \mathrm{~nm}$ deposited under the low Ar pressure condition are convenient for the hybrid recording media. These media have the strong exchange coupling between the magnetic grains. So, the wall pinning site density in the media must be increased in order to obtain a low noise media with a small domain size. The data will be probably recorded in the decoupled media at the lower temperature than that of the media with the strong exchange coupling.

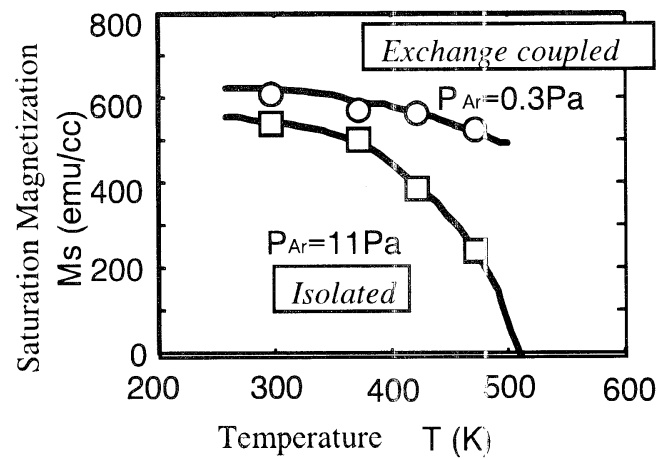

Fig.8 Temperature dependence of $M_{\mathrm{s}}$ for $\mathrm{Co} / \mathrm{Pd}$ specimens prepared by low $\mathrm{Ar}$ pressure deposition $(0.3 \mathrm{~Pa})$ and high pressure deposition $(11 \mathrm{~Pa})$.

\subsection{FePt and CoPt $\mathrm{L1}_{0}$ ordered alloys}

FePt and CoPt ordered alloy thin films with the $\mathrm{L}_{0}$ phase structure have been actively investigated for the perpendicular magnetic recording media with enhanced thermal stability. However, these media need very strong head field for magnetization reversal. Figure 9 shows the relationship between the remarent coercivity $H_{\mathrm{r}}$ of the CoPt ordered thin film in addition of B element and the pulse width of applying magnetic field. The specimen was deposited at room temperature by an electron cyclotron resonance sputtering method, and heat treatment carried out at a temperature of $600{ }^{\circ} \mathrm{C}$ after deposition. The $H_{\mathrm{r}}$ was measured by the custom-made vibrating sample magnetometer system equipped with a conventional electromagnet (maximum field $=18 \mathrm{kOe}$ ) and a pulse magnetic field generator (pulse width $=1 \mu \mathrm{s} \quad 1 \mathrm{~s}$, magnetic field $=0 \square 7.5 \mathrm{kOe}$ ). The $H_{0}$ is roughly estimated to be $20 \mathrm{kOe}$ by fitting of the data points to the Sharrock's equation ${ }^{28)}$. So, this result indicates that strong head field more than $20 \mathrm{kOe}$ is necessary to write in this medium at room temperature. As shown in Fig. 10, the coercivity of this specimen decreases with increasing the 
temperature. With heating up to $530 \mathrm{~K}$, the coercivity decreases to $1.2 \mathrm{kOe}$, which is only one-fifth of the coercivity at $300 \mathrm{~K}$, and the $H_{0}$ is supposed to be about 4 kOe. The data can be recorded on the CoPt ordered media by the conventional write head, when the media is heated. So, the $\mathrm{Ll}_{0}$ ordered alloys are useful for the thermal assisted media.

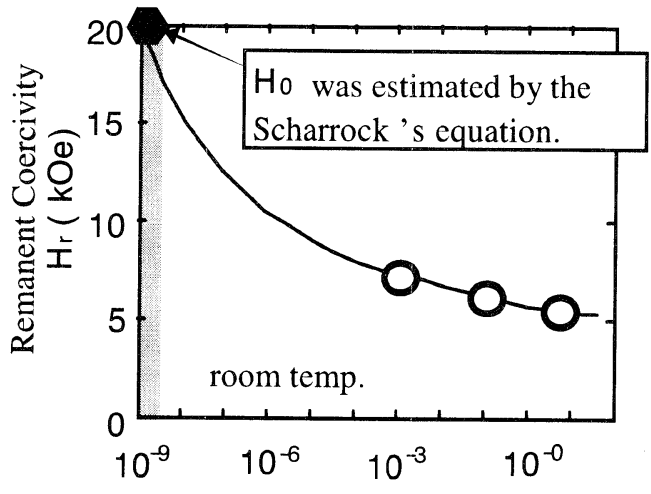

Magnetic Field Pulse width $\Delta t$ (sec)

Fig.9 Relationship between remanent coercivity $H_{\mathrm{r}}$ of the Co-Pt-B ordered thin film and the pulse width of applying magnetic field.

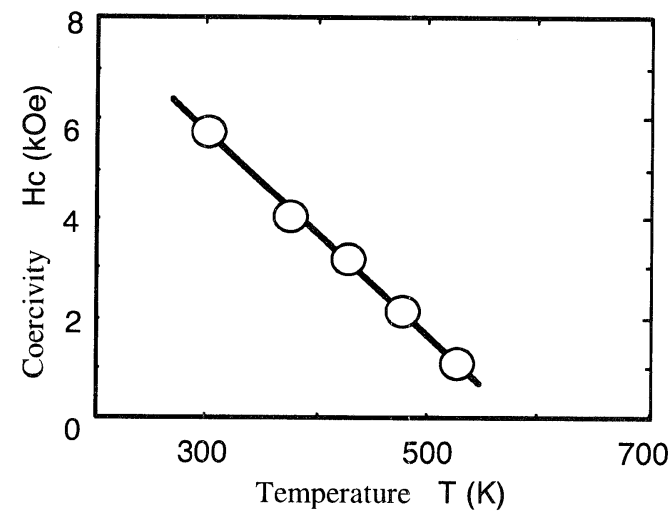

Fig.10 Temperature dependence of coercivity $H_{\mathrm{c}}$ for Co-Pt-B ordered thin film.

However, there are many problems which should be solved before practical application of the $\mathrm{Ll}_{0}$ ordered alloys. The major problems are

i) decrease of ordering temperature,

ii) reduction of medium noise.

The FePt and CoPt thin films need a heat treatment at a high temperature around $900 \mathrm{~K}$ during deposition and/or post-deposition annealing, in order to obtain the $\mathrm{L}_{0}$ ordered phase. The heat treatment temperature is about $300 \mathrm{~K}$ higher than the substrate temperature during the deposition of the conventional $\mathrm{Co}-\mathrm{Cr}-\mathrm{Pt}$ media on glass substrates. Since the glass transition temperature of the glass substrates is lower than the heat treatment temperature, the reduction of the ordering temperature is required for the development of the $\mathrm{L}_{0}$ ordered media. Some approaches have been proposed to reduce the ordering temperature of the $\mathrm{Ll}_{0}$ ordered alloys: e.g. the addition of a third elements such as $\mathrm{Cu}, \mathrm{B}$, and $\mathrm{Sb}^{28)-30 \text { ), }}$ employment of a multilayered $\mathrm{Fe} / \mathrm{Pt}$ films $^{31)}$, and sputter-deposition under an extremely high gas pressure condition $^{32}$. Figure 11 shows the relationship between the annealing temperature after deposition and the coercivity of the FePt specimens prepared by the above-mentioned three methods. The coercivity of those three specimens increase at the annealing temperature between $600 \mathrm{~K}$ and $700 \mathrm{~K}$, that means the ordering temperature of those specimens is decreased by about $200 \mathrm{~K}$. This result suggests that the FePt and the CoPt ordered alloy media can be deposited on the glass substrates.

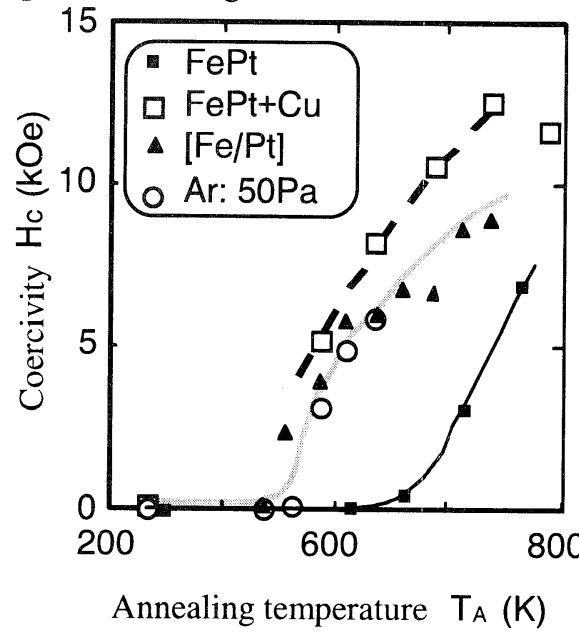

Fig.11 Relationship between annealing temperature after deposition and $H_{\mathrm{c}}$ of FePt specimens ${ }^{28), 31), 32) \text {. }}$

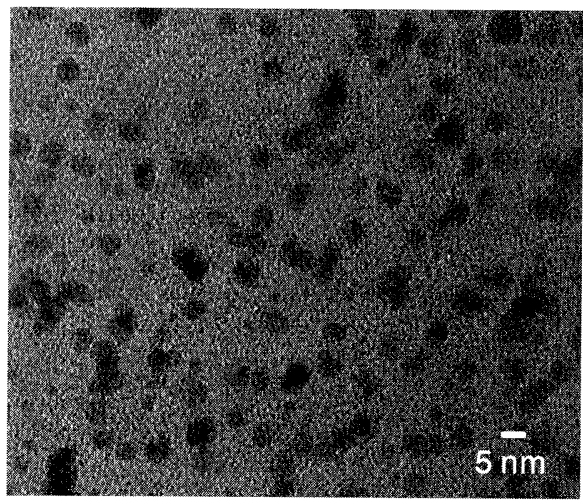

Fig.12 Transmission electron microscope (TEM) image of chemically synthesized FePt $\mathrm{L}_{0}$ ordered particles $^{35)}$.

The other problem is the reduction of the medium noise. In order to decrease the medium noise, the magnetic switching volume of the FePt media must be reduced by breaking the exchange coupling between the FePt grains. Granular media and film formed by chemical reaction have been studied for reducing the exchange interaction. The granular media are $\mathrm{FePt}$ composite films with a non-magnetic matrix such as $\mathrm{SiO}_{2}{ }^{36)}, \mathrm{MgO}^{37)}, \mathrm{Al}_{2} \mathrm{O}_{3}, \mathrm{ZrO}^{9)}$, $\mathrm{B}_{2} \mathrm{O}_{3}{ }^{38)}, \mathrm{TaN}, \mathrm{Si}_{3} \mathrm{~N}_{4}, \mathrm{Ag}, \mathrm{B}^{39)}$, and $\mathrm{C}^{40)}$. The switching volume is decreased with adding the matrix up to $30 \mathrm{vol} . \%$, since the non-magnetic matrix isolate the FePt crystal grains. The granular media are effective to the reduction of 
the medium noise, however it is difficult to decrease the ordering temperature of the $\mathrm{FePt}$ crystal grains. Chemically synthesized FePt fine particles with the $\mathrm{Ll}_{0}$ ordered structure have been also studied for the high density recording media with the low medium noise ${ }^{34)}$. Figure 12 shows the TEM image of the $\mathrm{L1}_{0}$ ordered FePt particles synthesized with the reduction method by using an ethylene glycol $^{35)}$. The coercivity of the particles is more than $9 \mathrm{kOe}$, though the grain size is only about $5 \mathrm{~nm}$.

\section{Conclusions}

Increase of medium switching field and head field limit put the limit on the use of high $K_{\mathrm{u}}$ materials for high areal density magnetic recording. Since the hybrid recording solve the head field limit problem, the hybrid recording media will be able to use extremely high $K_{\mathrm{u}}$ materials, such as $\mathrm{FePt}$ and $\mathrm{CoPt} \mathrm{L1}_{0}$ ordered alloy permanent magnet materials, $\mathrm{Co} / \mathrm{Pt}$ and $\mathrm{Co} / \mathrm{Pd}$ multilayer thin films, and $\mathrm{Tb}-\mathrm{Fe}-\mathrm{Co}$ magneto optical recording media, whose $K_{\mathrm{u}}$ values are more than $10^{7} \mathrm{erg} / \mathrm{cc}$. The bit transitions are determined by the heating profile of the media as well as the head field gradient. Therefore, tiny bit patterns will be probably recorded in the strong exchange coupling media. The hybrid recording media have "huge" alternative of not only high $K_{\mathrm{u}}$ materials but also the film structure.

Acknowledgements Authors would like to great thank to colleagues of Hitachi Maxell Ltd., Dr. Teruaki Takeuchi, Dr. Satoshi Matsunuma, Mr. Hideo Daimon, and Dr. Hiroyuki Awano, for their contribution to this work.

\section{References}

1) R. Wood: IEEE Trans. Magn., 36, 36 (2000).

2) R. Wood, J. Miles, and T. Olson: IEEE Trans. Magn., 38, $1711(2002)$

3) Z. Jin, H. Bertram, B. Wilson, and R. Wood: IEEE Trans. Magn., 38, 1429 (2002).

4) J. Miles, D. McKirdy, R. Chantrell, and R. Wood: IEEE Trans. Magn., 39, 1876 (2003).

5) M. Mallary, A. 'Torabi, and M. Benakli: IEEE Trans. Magn., 38, 1615 (2002).

6) H. Bertram, H. Zhu, and R. Gustafson: IEEE Trans. Magn., 34, 1845 (1998).

7) S. Charap, P. Lu, and Y. He: IEEE Trans. Magn., 33, 978 (1997).

8) D. Weller and A. Moser: IEEE Trans. Magn., 35, 4423 (1999).

9) K. Coffey, M. Parker, and J. Howard: IEEE Trans. Magn., 31, 2737 (1995).

10) N. Inaba, Y. Uesaka, and M. Futamoto: IEEE Trans. Magn., 36, 54 (2000).

11) Y. Kanai, R. Matsubara, H. Watanabe, H. Muraoka, and Y. Nakamura: IEEE Trans. Magn., 39, 1955 (2003).

12) T. Rausch, P. Herget, J. Bain, J. Zhu, D. Stancil and T. Schlesinger: Proceeding of SPIE, 4342, 502 (2002).

13) M. Alex, A. Tselikov, T. McDaniel, N. Deeman, T. Valet, and D. Chen: IEEE Trans. Magn., 37, 1244 (2001).

14) J. Ruigrok: J. Magn. Soc. Japan, 25, 313 (2001).
15) M. Alex, T. Valet, T. McDaniel, and C. Brucker: J. Magn. Soc. Japan, 25, 328 (2001).

16) T. McDaniel, W. Challener, and K. Sendur: IEEE Trans. Magn., 39, 1972 (2003).

17) K. Kojima, M. Hamamoto, J. Sato, K. Watanabe, and H. Katayama: IEEE Trans. Magn., 37, 1406 (2001).

18) T. Takeuchi (unpublished).

19) R. Kikuchi: J. Appl. Phys., 27, 1352 (1956).

20) Q. Peng and H. Bertram: IEEE Trans. Magn., 33, 2677 (1997).

21) M. Benakli, A. Torabi, M. Mallary, H. Zhou, and H Bertram: IEEE Trans. Magn., 37, 1564 (2001).

22) N. Inaba, Y. Uesaka, A. Nakamura, M. Futamoto, Y. Sugita, and S. Narishige: IEEE Trans. Magn., 33, 2989 (1997).

23) J. Hohlfeld, Th. Gerrits, M. Bilderbeek, Th. Rasing, H. Awano, and N. Ota: Phys. Rev. B, 65, 012413 (2001).

24) H. Awano, S. Imai, M. Sekine, M. Tani, N. Ota, K. Mitani, N. Takagi, H. Noguchi, and M. Kume: IEEE Trans. Magn., 36, 2261 (2000).

25) K. Kojima, M. Hamamoto, J. Sato, K. Watanabe, and H. Katayama: IEEE Trans. Magn., 37, 1406 (2001).

26) H. Kerseren and W. Zeper: J. Magn. Magn. Mats., 120, 271 (1993).

27) W. Zeper, F. Greidanus, and P. Carcia: IEEE Trans. Magn., 25, 3764 (1989).

28) M. Sharrock: J. Appl. Phys., 76, 6314 (1994).

29) O. Kitakami, S. Okamoto, N. Kikuchi, T. Miyazaki, and Y. Shimada: J. Magn. Soc. Japan., 26, 1047 (2002).

30) O. Kitakami, Y. Shimada, K. Oikawa, H. Daimon, and K. Fukamichi: Appl. Phys. Lett., 78, 1104 (2001).

31) T. Maeda, A. Kikitsu, T. Kai, T. Nagase, H. Aikawa, and J. Akiyama: IEEE Trans. Magn., 38, 2796 (2002).

32) Y. Endo, N. Kikuchi, O. Kitakami, and Y. Shimada: J. Appl. Phys., 89, 7065 (2001).

33) T. Suzuki: Materials Trans., 44, 1535 (2003).

34) S. Sun, C. Murray, D. Weller, L. Folks, and A. Moser: Nature, 287, 1989 (2000).

35) Y. Kurobe, H. Daimon, N. Ota, and N. Toshima: The Bulletin of the Nano Science and Technology, 1, 15 (2003). (in Japanese)

36) C. Luo and D. Sellmyer: Appl. Phys. Lett., 75, 3162 (1999).

37) T. Suzuki and K. Ouchi: IEEE Trans. Magn., 37, 1283 (2001).

38) C. Luo, S. Liou, L. Gao, Y. Liu, and D.Sellmyer: Appl. Phys. Lett., 7, 2225 (2000).

39) N. Li and B. Lairson: IEEE Trans. Magn., 35, 1077 (1999).

40) M. Yu, Y. Liu, and D. Sellmyer: J. Appl. Phys., 87, 6959 (2000).

Received June 3, 2004 ; Accepted June 16, 2004 